\title{
Neue Möglichkeiten zur Ermittlung der Nähr- und Schadstoffbelastung in kleinen Fließgewässern - Stationärer Organik- und Anorganik-Sammler
}

\author{
Peter Flödl · Sophie Stelzer · Ottavia Zoboli · Arabel Aman · Tobias Mayer · Matthias Zessner · Christoph Hauer
}

Online publiziert: 22. Januar 2020

(C) Der/die Autor(en) 2020

Zusammenfassung Die anthropogen verursachte Schadstoffbelastung stellt für das Ökosystem ein potenzielles Risiko dar, weshalb in der EU-Wasserrahmenrichtlinie Zielzustände definiert wurden, um eine nachhaltige Nutzung von Oberflächengewässern auch zukünftig zu sichern. Die hohe Anzahl an Schadstoffen, die oft auch nur in sehr geringen Konzentrationen in der aquatischen Umwelt auftreten, erschweren jedoch deren Nachweis. Die Erforschung der Eintragspfade in Fließgewässer, die Schadstoffmobilität sowie deren mögliche Folgen stellen daher eine besondere Herausforderung dar. Für die Ermittlung der auftretenden Nährstoffe, Schwermetalle und Spurenstoffe wurden deshalb unterschiedliche Messgeräte und Probenahmetechniken entwickelt, die eine Beprobung der Schwebstoffe, der Sedimente und des Oberflächenwassers ermöglichen. Hierbei zeigen sich je nach Methode Vorund Nachteile, wobei oft eine Limitierung der Anwendbarkeit auf mittlere bis große Gewässer besteht. Für die Ermittlung der Schadstoffkonzentrationen in kleinen Gewässern mit geringen Fließgeschwindigkeiten gibt es hingegen nur eine kleine Auswahl an Messmethoden,

DI P. Flödl (凶) • DI S. Stelzer, BSc • DI T. Mayer · PD DI Dr. C. Hauer Department für Wasser - Atmosphäre - Umwelt, Christian Doppler Labor für Sedimentforschung und -management, Institut für Wasserbau, Hydraulik und Fließgewässerforschung, Universität für Bodenkultur Wien, Muthgasse 107, 1190 Wien, Österreich peter.floedl@boku.ac.at

Dr. O. Zoboli, MSc · DI A. Aman, BSc . Univ.-Prof. DI Dr. M. Zessner Institut für Wassergüte und Ressourcenmanagement, Technische Universität Wien, Karlsplatz 13, 1040 Wien, Österreich die jedoch meist eine gute Infrastruktur vor Ort benötigen. In diesem Beitrag wird gezeigt, dass eine adaptierte Version eines mobilen Geschiebefängers zum Sammeln einer ausreichenden Menge Feinmaterial (Korngrößen $<1 \mathrm{~mm}$ ) sowie Schwimmstoffe genutzt werden kann, um diese anschließend im Labor auf Schadstoffkonzentrationen untersuchen zu können. Die ersten Untersuchungen zeigen hierbei interessante Erkenntnisse in Bezug auf die Verteilung der Schadstoffkonzentrationen und auf das Forschungspotenzial im Bereich des Schadstoffeintrags und -transports in kleinen Einzugsgebieten $\left(<100 \mathrm{~km}^{2}\right)$

Schlüsselwörter Schadstoffe . Spurenstoffe · Fließgewässer - Passive Schwimm- und Schwebstoffsammlung

New possibilities to determine nutrient and pollutant loads in small streams - Stationary organic and inorganic sampler

Abstract Anthropogenic pollution causes a potential risk for the ecosystem, hence the EU-Water Framework Directive defines quality standards to ensure the sustainable use of surface waters in the future. However, the high number of pollutants, which often occur in the aquatic environment even in very low concentrations, makes their detection difficult. Research is therefore particularly challenging with regard to the input paths, the mobility of pollutants and their possible consequences in aquatic ecosystems. For determination of the occurring nutrients, heavy metals and trace substances, different measuring instruments and sampling techniques have therefore been developed, which allow the sampling of suspended solids, sediments and surface water. Depending on the method used, there are advantages and disadvantages, although the applicability is often limited to medium to large rivers. For determination of pollutant concentrations in small streams with low flow velocities, however, there is only a small selection of measuring methods available, which usually require a good infrastructure on site. In this paper it could be shown that an adapted version of a mobile bed-load sampler can be used to collect fine material (grain sizes $<1 \mathrm{~mm}$ ) as well as floating material in sufficient quantity to be able to examine these afterwards in a laboratory for pollutant concentrations. The first investigations show interesting findings regarding the distribution of pollutant concentrations and the research potential in the field of pollutant input and transport in small catchment areas $\left(<100 \mathrm{~km}^{2}\right)$.

Keywords Pollutants · Trace substances · Rivers · Passive collection of floating and suspended matter

\section{Einleitung}

Die anthropogen verursachte Schadstoffbelastung der Fließgewässer, Seen und Meere stellt weltweit nicht nur für aquatische Lebewesen ein potenzielles Risiko dar (Malaj et al. 2014). Durch die Belastung von Trinkwasserressourcen und die Akkumulation von Schadstoffen in Fischen gelangen diese unter anderem auch in den Nahrungskreislauf des Menschen (Järup 2003; Shinn et al. 2009). Die europäischen Mitgliedstaaten haben deshalb in der EUWasserrahmenrichtlinie (2000/60/EG) Zielzustände definiert, um eine nachhaltige Nutzung von Oberflächengewässern sicher zu stellen. Mit der Novellierung der Richtlinie 2008/105/EG wurden 33 prioritäre Stoffe und 8 weitere Schadstoffe festgelegt, mit denen der gute chemische Zustand eines Gewässers festzustellen ist. Jedoch können deutlich mehr Stoffe in der aquatischen Umwelt festgestellt werden, die über- 


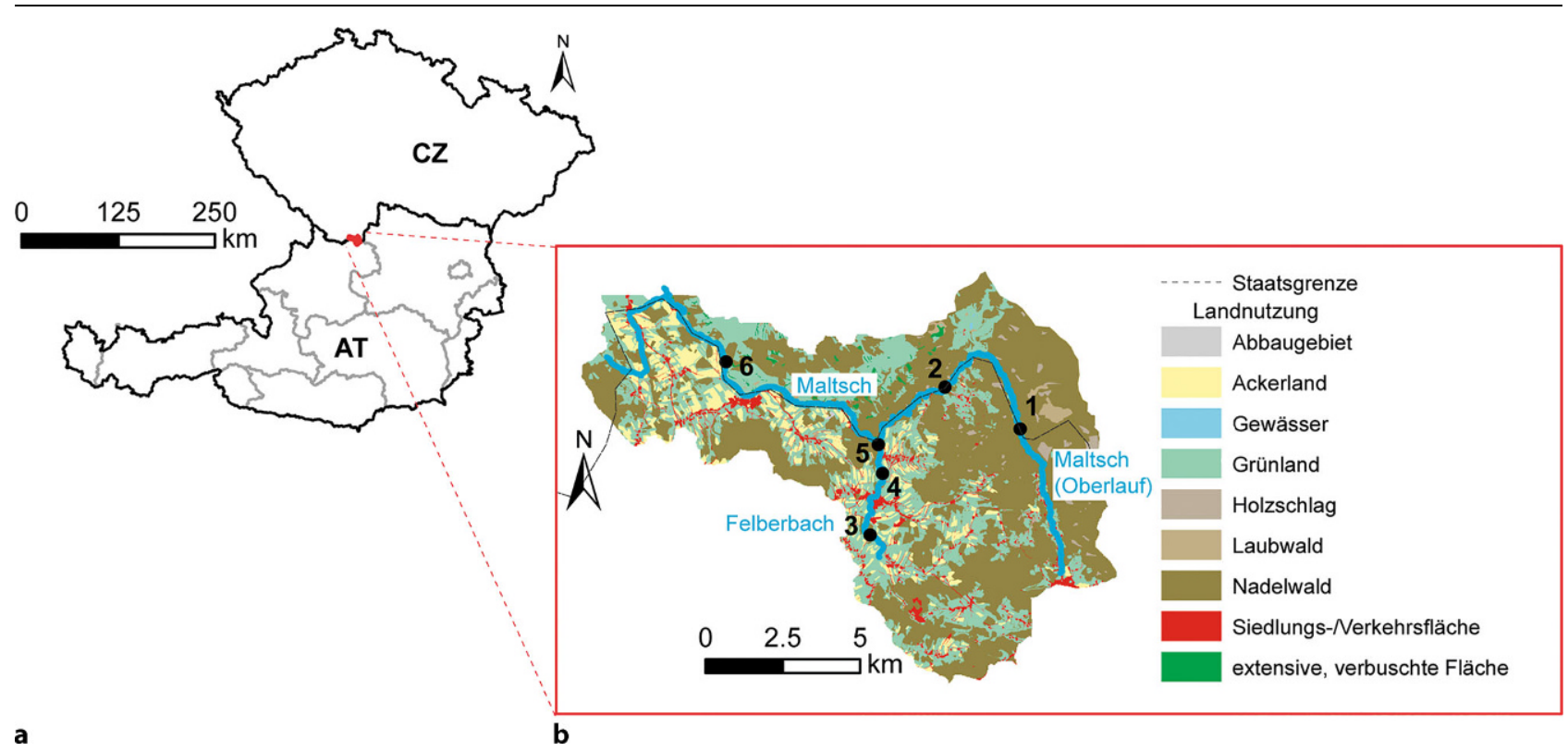

Abb. 1 a Übersicht über das Projektgebiet (rote Markierung): Das Einzugsgebiet der Maltsch erstreckt sich über Österreich und Tschechien. b Detaillierte Darstellung des Einzugsgebiets: Die Probenahmestellen (Nr. 1-6) befinden sich an der Maltsch und dem wichtigsten Zubringer (Felberbach)

geordnet als Spurenstoffe organische Substanzen umfassen. Diese können bereits in sehr geringen Konzentrationen (ng/l bis $\mu \mathrm{g} / \mathrm{l}$ ) negative Wirkungen auf Menschen und Tiere haben (ÖWAV 2013). Als wesentliche Quellen finden sich in publizierten Studien unter anderem Bergbauaktivitäten, die Schwerindustrie, Kläranlagenabläufe sowie Substanzen aus der intensiven Landwirtschaft (Garcia-Ordiales et al. 2017; Zessner et al. 2019; Zoboli et al. 2019). Der Eintrag in die aquatische Umwelt kann hierbei direkt (punktuell) oder diffus erfolgen (Clara et al. 2014; Schwarzenbach et al. 2010). Im Gewässer angelangt, werden Spurenstoffe und Schwermetalle in der Wassersäule gelöst und partikulär oder an gelöste organische Substanz gebunden transportiert (Haitzer et al. 1998; Inostroza et al. 2017). Die hohe Anzahl an Spurenstoffen und Schwermetallen, geringe Konzentrationen und unterschiedliche Bindungsaffinitäten stellen hierbei eine besondere Herausforderung bei der Bestimmung dar (ÖWAV 2013). Für die Ermittlung der Schadstoffe in Fließgewässern können unterschiedliche Medien (z.B. Wasser, Sediment, Schwebstoffe etc.) untersucht werden, wobei sich Limitierungen über eine generelle Aussage der vorherrschenden Schadstoffbelastung aus dem jeweiligen Konzept der Probenahme ergeben. Wasserproben aus der fließenden Welle eines Fließgewässers stellen eine Momentaufnahme dar und erfassen als solche die Schadstoffkonzentration nur zu einem bestimmten Zeitpunkt. Für eine repräsentative Erfassung der eingebrachten Spurenstoffe und Schwermetalle ist es jedoch notwendig, abhängig vom jeweiligen Niederschlagsereignis automatisch mengenproportionale Proben zu entnehmen, um die Variabilität der Belastung zu erfassen (Clara et al. 2014). Die Entnahme einer abgelagerten Sedimentprobe repräsentiert die Akkumulation von Schadstoffen über einen nicht näher definierbaren Zeitraum, wodurch Umwandlungs-, und Abbauprozesse einen schwer zu quantifizierenden Faktor darstellen (z.B. Quecksilber-Methylierung; Avramescu et al. 2011; Ouddane et al. 2014). Des Weiteren zeigt sich eine (hohe) Variabilität der Belastung in Abhängigkeit vom Entnahmepunkt aufgrund morphologischer und hydrologischer Einflüsse (Förstner 2004). Für die Erfassung der an Schwebstoffe gebundenen (und transportierten) Schadstoffe gibt es unterschiedliche passive Probensammler (u. a. Draxler et al. 2012; Phillips et al. 2000), die sich insbesondere für mittlere bis große Fließgewässer eignen. Hierbei zeigen sich jedoch bei der Beprobung von Schwebstoffen in Fließgewässern mit geringen Fließgeschwindigkeiten und hoher organischer Belastung Probleme durch (biologische) Kolmation.
Auch im Zuge des Interreg-Projekts zur Förderung der natürlichen Umwelt und des Vorkommens der Flussperlmuschel im Maltsch-Einzugsgebiet führte die biologische Kolmation zu Problemen bei der ursprünglich geplanten Geschiebesammlung. Auf Basis erster Beobachtungen und Messungen sollte untersucht werden, ob die eingesetzten Sammler für die Erfassung von organischem und anorganischem Material bei geringen Durchflüssen verwendet werden können und somit für die Ermittlung von Spurenstoff- und Schwermetallkonzentrationen in den Schwebstoffen einsetzbar sind. In diesem Beitrag werden erste Erkenntnisse und Erfahrungen im Einzugsgebiet der Maltsch vorgestellt und potenzielle Anwendungsgebiete für die Beurteilung von Nähr- und Schadstoffbelastungen in kleinen Fließgewässern aufgezeigt.

Untersucht wurden folgende Aspekte:

- Sammlung von organischen und anorganischen Schweb- und Schwimmstoffen über einen definierten Zeitraum ( $24 \mathrm{~h}$ bis 2 Wochen), trotz geringer Fließgeschwindigkeiten und geringer Durchflüsse.

- Unterschiede in der Schadstoffkonzentration zwischen abgelagertem Material (unbekannte Ablagerungszeit) im Vergleich zu dem Sammelgut im vorgestellten SOA(stationärer 

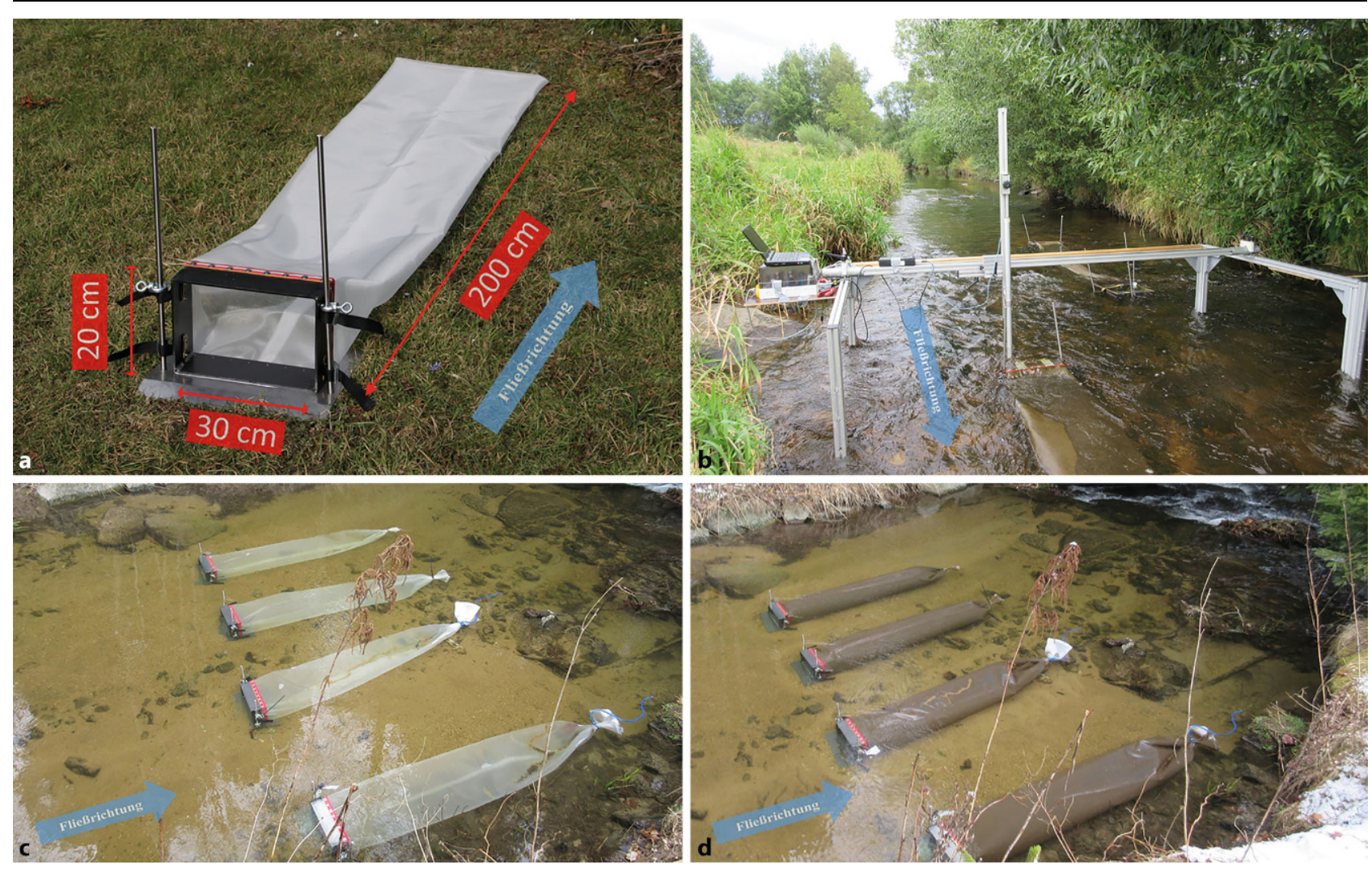

Abb. 2 a Adaptierter Geschiebesammler nach Bunte et al. (2007) und b Messungen der Fließgeschwindigkeiten im Nahbereich der Sammler mittels zylindrischer Flügelradsonde (Durchmesser $25 \mathrm{~mm}$ ). Fotos der ersten Tests zur Sammelkapazität: c aufgenommen nach Einbau der Sammler und $\mathbf{d}$ Zustand $24 \mathrm{~h}$ nach dem Einbau

Organik- und Anorganik)-Sammler (2-wöchige Expositionszeit).

- Unterschiede in den Schadstoffkonzentrationen zwischen den Teileinzugsgebieten.

\section{Methoden}

In den folgenden Abschnitten wird das Konzept zur Sammlung von organischen und anorganischen Schweb-, und Schwimmstoffen vorgestellt, weiters werden die Methoden zur Untersuchung hinsichtlich Sammelkapazität beschrieben.

\subsection{Beschreibung des Einzugsgebiets und der Probenahmestellen}

Das Untersuchungsgebiet befindet sich im nördlichen Mühlviertel im Einzugsgebiet der Maltsch (Elbe-Einzugsgebiet). Das rund $120 \mathrm{~km}^{2}$ große Einzugsgebiet gliedert sich in drei große Teileinzugsgebiete: (i) Maltsch Oberlauf $\left(38 \mathrm{~km}^{2}\right)$, (ii) Felberbach $\left(37 \mathrm{~km}^{2}\right)$ und (iii) das unterhalb der Felberbachmündung gelegene Maltsch-Teileinzugsgebiet $\left(45 \mathrm{~km}^{2}\right)$. In den beiden oberen
Teileinzugsgebieten zeigen sich deutliche Unterschiede hinsichtlich Geologie, Gefälle und Landnutzung (Abb. 1). Das Maltsch-Teileinzugsgebiet bis zum Felberbachzufluss ist mit einem Flächenanteil von $76 \%$ von Fichtenwäldern dominiert. Der Felberbach, dessen Einzugsgebiet in etwa die gleiche Fläche wie die Maltsch bis zum $\mathrm{Zu}$ sammenfluss aufweist, kommt aus dem Feinkorngranit und bildet sanftere Hügel, die vorwiegend zur Grünland- und Ackerbewirtschaftung genutzt werden. Flussab des Felberbachzuflusses nehmen die Ackerflächen weiter zu.

Die SOA- (stationäre Organik- und Anorganik-) Sammler wurden im Juni 2019 für zwei Wochen an repräsentativen Abschnitten in der Maltsch ( $n=2$ Sammler) und im Felberbach $(n=3 \quad$ Sammler $)$ installiert. Am 26.06.2019 wurden dann jeweils Proben aus dem Sammler und dem abgelagerten Sediment im Nahbereich entnommen. Die Proben aus den Sammlern repräsentieren hierbei die Schwebstoffe und die entsprechenden Spurenstoffund Schwermetallgehalte an diesen Schwebstoffen, die über einen Zeit- raum von zwei Wochen transportiert wurden. Über jene Sedimentproben, die im Nahbereich der Sammler entnommen wurden, können keine Angaben zur Dauer der Ablagerungszeit gegeben werden. Sie repräsentieren die Schadstoffkonzentrationen, die sich über einen längeren Zeitraum im Sediment akkumulieren.

\subsection{Funktionsweise des Stationären Organik- und Anorganik-Sammlers (SOAS)}

Der Sammler basiert auf dem Konzept des mobilen Geschiebefängers von Bunte et al. (2007), welcher ursprünglich für die Quantifizierung des Geschiebetransports in watbaren Fließgewässern entwickelt wurde. Für das Interreg-Projekt wurde er für die Sammlung von Feinmaterial (Korngrößen $<1 \mathrm{~mm}$ ) und Schwimmstoffe optimiert. Der Fänger besteht aus einem Stahlrahmen $(0,2 \times 0,3 \mathrm{~m})$, der auf eine Bodenplatte gestellt und mit zwei Stangen im Flussbett befestigt wird (Abb. 2a). Die Sammlung erfolgt in einem $2 \mathrm{~m}$ langen Netz, das eine Maschenweite 


\begin{tabular}{|c|c|c|c|c|c|}
\hline Parameter & Analysemethode & $\begin{array}{l}\text { Verwendetes } \\
\text { Gerät }\end{array}$ & Bestimmungsgrenze & Nachweisgrenze & Einheit \\
\hline Blei & \multirow[t]{6}{*}{ EPA Method 6020A:2007 } & \multirow[t]{6}{*}{ Agilent 7900 ICP-MS 01} & 0,01 & 0,005 & $\mathrm{mg} / \mathrm{kg}$ TS \\
\hline Cadmium & & & 0,01 & 0,005 & $\mathrm{mg} / \mathrm{kg}$ TS \\
\hline Kupfer & & & 0,02 & 0,01 & $\mathrm{mg} / \mathrm{kg}$ TS \\
\hline Nickel & & & 0,01 & 0,005 & $\mathrm{mg} / \mathrm{kg}$ TS \\
\hline Quecksilber & & & 0,02 & - & $\mathrm{mg} / \mathrm{kg}$ TS \\
\hline Zink & & & 0,1 & 0,05 & $\mathrm{mg} / \mathrm{kg}$ TS \\
\hline PFOA & \multirow[t]{2}{*}{ WBSE-121:2019 } & \multirow[t]{2}{*}{ 1290_HPLC_6495_QQQ } & 0,002 & 0,001 & $\mu \mathrm{g} / \mathrm{kg}$ TS \\
\hline PFOS & & & 0,002 & 0,001 & $\mu g / \mathrm{kg}$ TS \\
\hline
\end{tabular}

Tab. 2 Fließgeschwindigkeitsänderungen durch die (biologische) Kolmation der Netze. Gemessen wurden die Geschwindigkeiten oberflächennahe vor und nach Reinigung der Netze

\begin{tabular}{|c|c|c|c|c|}
\hline $\begin{array}{l}\text { Position vor } \\
\text { dem Sammler }\end{array}$ & $\begin{array}{l}\text { Fließgeschwindigkeit } \\
\text { ohne Verlegung }(\mathrm{m} / \mathrm{s})\end{array}$ & $\begin{array}{l}\text { Fließgeschwindigkeit } \\
\text { mit Verlegung }(\mathrm{m} / \mathrm{s})\end{array}$ & $\begin{array}{l}\text { Reduktion der Fließgeschwindig- } \\
\text { keit }(\mathrm{m} / \mathrm{s})\end{array}$ & $\begin{array}{l}\text { Reduktion der Fließgeschwin- } \\
\text { digkeit (\%) }\end{array}$ \\
\hline Links & 0,30 & 0,24 & 0,06 & 19 \\
\hline Mitte & 0,31 & 0,24 & 0,07 & 22 \\
\hline Rechts & 0,31 & 0,24 & 0,07 & 22 \\
\hline
\end{tabular}

von $0,5 \mathrm{~mm}$ aufweist. Das Ende wird mittels Kabelbinder verschlossen und ermöglicht so eine Entnahme des Sammelguts sowie gute Reinigungsmöglichkeit. Nach Befestigung des Sammlers im Flussbett kann Wasser durch das Netz strömen, transportiertes organisches und anorganisches Material wird hingegen zurückgehalten. Ziel dieser Sammelmethode ist, dass durch die fortschreitende (biologische) Kolmation des Netzes die Sammelleistung ansteigt, da immer kleinere Partikel rückgehalten werden können (Abb. 2c,d). Für die Ermittlung eines möglichen Rückstaueffekts durch die Verlegung des Netzes (und einer damit möglichen Abnahme der Sammelleistung) wurden Fließgeschwindigkeiten oberflächennahe $(80 \%$ der Wassertiefe) gemessen. Hierfür wurden die Sammler für drei Tage in einem repräsentativen Gewässerabschnitt installiert und Fließgeschwindigkeiten im Nahbereich des Sammlereinlaufs, links, mittig und rechts gemessen (Abb. 2b). Die Referenzmessung erfolgte nach Entleerung und Reinigung der Sammler direkt anschließend an die erste Messung an den gleichen Punkten. Die Geschwindigkeitsmessung wurde mit einem Labor-Messflügel (Flügeldurchmesser $=25 \mathrm{~mm}$ ) der Firma Höntzsch (SONDE ZS25 ... ZG1) durchgeführt. Für die exakte Positionierung der Messsonde kam ein $1,5 \times 2,5 \mathrm{~m}$ großer Aluminiumrahmen zum Einsatz, auf dem der Flügel exakt in X-, Y- und Z-Richtung ausgerichtet werden kann. Hierdurch wurde sichergestellt, dass Positionsfehler minimiert werden und die Fließgeschwindigkeitsmessungen gut vergleichbar sind. Mittels der Software DASYlab wurde jede Einzelmessung $30 \mathrm{~s}$ lang aufgezeichnet und der Mittelwert berechnet.

\subsection{Chemische Analysen}

Die aus dem adaptierten Geschiebesammler nach Bunte et al. (2007) gezogenen Schwebstoffproben sowie die aus Ablagerungen gewonnenen Feinsediment-Proben wurden möglichst rasch ins Labor transportiert und dort bis zur Weiterverarbeitung am nächsten Tag gekühlt gelagert. Probe 1, die stark durch altes Laub geprägt war, wurde vor der Weiterverarbeitung homogenisiert. Die Trockenmasse bzw. Trockensubstanzgehalt (TS) und Glühverlust bzw. der Gehalt an organischer Trockensubstanz (oTS) aller Proben wurde im Labor des Instituts für Wassergüte und Ressourcenmanagement mit Standardmethoden analysiert. $200 \mathrm{~g}$ jeder Probe wurden gekühlt und mit Expressservice zu WESSLING Hungary Kft., einem akkreditierten Labor für die Analyse organischer und anorganischer Spurenstoffe in Wasser- und Sedimentproben, geliefert. Der Parameterumfang bei der Analyse umfasste die Schwermetalle Blei, Cadmium, Kupfer, Nickel, Quecksilber und Zink sowie PFOS (Perfluoroctansulfonsäure) und PFOA (Perfluoroctansäure) als Vertreter der Gruppe der per- und polyfluorierten Alkylverbindungen (PFAS). Dies ist eine Gruppe von Stoffen mit vielfältigem Einsatzmuster wie z. B. in Farben, Leder- und Textilbeschichtungen, (Outdoor-)Kleidung, Schuhen, Teppi- chen, Verpackungen, Skiwachs, Bodenund Autopflegemitteln sowie zur Produktion von Papieren mit schmutz-, fett- und wasserabweisenden Eigenschaften und als Bestandteile von Imprägnier- und Schmiermitteln. PFOS und PFOA werden über den Wasserpfad, aber auch über den Luftpfad in der Umwelt verteilt. In Tab. 1 sind die Standardmethoden, nach denen die Analysen durchgeführt wurden, die verwendeten Geräte und die Bestimmungs- sowie Nachweisgrenzen für den jeweiligen Parameter dargestellt.

\section{Ergebnisse}

Wie in Abb. 2c, d zu sehen, konnte bereits im ersten Testlauf nach $24 \mathrm{~h}$ eine Teilverlegung der Netze festgestellt werden und organisches und anorganisches Material aus den Sammlern entnommen werden. Der Einfluss der Verlegung auf die Fließgeschwindigkeiten wurde deshalb unter Naturbedingungen in einem weiteren Schritt getestet und es zeigte sich, dass im Nahbereich des Sammlereinlaufs eine gleichmäßige Reduktion auf linker, mittiger und rechter Seite stattfindet. Die Geschwindigkeitsreduktionen betrugen hierbei bis zu $0,07 \mathrm{~m} / \mathrm{s}$ bzw. $22 \%$ (Tab. 2 ), wobei die kritische Fließgeschwindigkeit nach Hjulström (1935) für Korngrößen $<63 \mu \mathrm{m}$ nicht unterschritten wird. Die Funktionsfähigkeit zum Sammeln des Materials scheint somit über $24 \mathrm{~h}$ gegeben zu sein. Dies hat sich auch daran gezeigt, dass sehr kleine Fraktionen aus abgelagerten Schweb- und Schwimmstoffen im Sammler vorzufinden waren. 

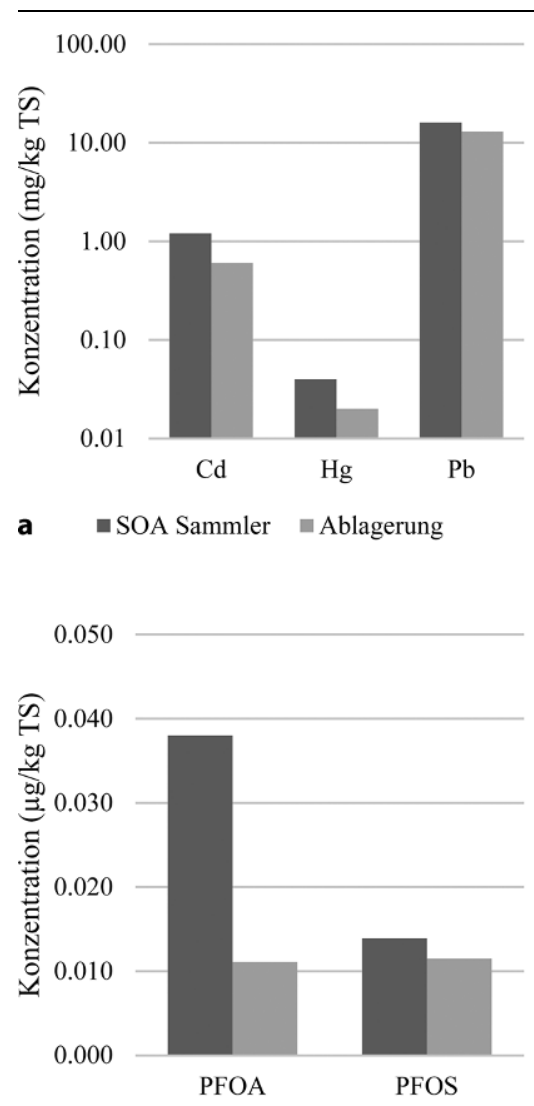

c $\quad$ SOA Sammler $\square$ Ablagerung
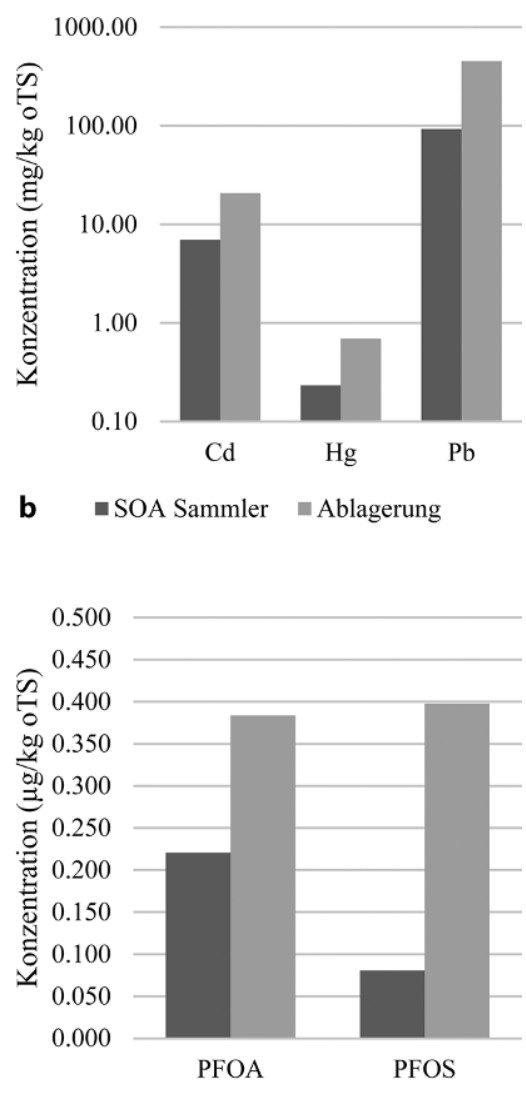

d $\quad$ SOA Sammler $\quad$ Ablagerung

Abb. 3 Konzentrationen der untersuchten Proben aus dem SOA-Sammler und den abgelagerten Sedimenten bezogen auf die Trockensubstanz (a, c) und die organische Trockensubstanz (b, d). Die Schwermetallkonzentrationen $(\mathbf{a}, \mathbf{b})$ sind in logarithmischer Skalierung aufgetragen; die Spurenstoffkonzentrationen (c, d) in linearer Skalierung

Die Analysen hinsichtlich der Unterschiede zwischen den Schadstoffkonzentrationen im Sammelgut des SOA-Sammlers im Vergleich zu den abgelagerten Sedimenten weisen deutliche Unterschiede auf. Diese können anhand der Schwermetallkonzentrationen für Cadmium, Quecksilber und Blei gezeigt werden (Probe 4, Abb. 3). Die Schwermetallkonzentrationen bezogen auf die Trockensubstanz sind im abgelagerten Feinsediment um 19 bis $50 \%$ kleiner als im Material des SOASammlers (Abb. 3). Die Konzentration der Schwermetalle bezogen auf die organische Fraktion ist hingegen im abgelagerten Material deutlich höher (bis zu $80 \%$ ), da der organische Anteil durch Klassierung bei der Ablagerung in den abgelagerten Feinsedimenten deutlich geringer ist als in den mit dem SOASammler gesammelten Schwebstoffen (Abb. 3). Die Untersuchungen auf Vertreter der Gruppe der per- und polyfluorierten Alkylverbindungen (PFAS) in der Trockensubstanz zeigen noch deutlichere Unterschiede der Konzentrationen ( 17 bis $71 \%$ ) zwischen dem abgelagerten Sediment und den Proben aus dem SOA-Sammler (Abb. 3, PFOA und PFOS). Konzentrationen bezogen auf die organische Trockensubstanz weisen ebenfalls Unterschiede zwischen den Probenahmetechniken auf. Diese betragen zwischen 43 und $80 \%$.

Die einzugsgebietsbezogenen Unterschiede können auf Basis der Proben aus dem SOA-Sammler dargestellt werden und repräsentieren die Schadstoff- und Spurenstoffkonzentrationen, die über zwei Wochen transportiert werden. Es zeigen sich für die untersuchten Schwermetalle in Bezug auf die Trockensubstanz die geringsten Belastungen unterhalb der Kläranlage (Felberbach, Probe 4; Abb. 4), sowie im Sammler kurz vor der Einmündung des Felberbachs in die Maltsch (Probe 5 ; Abb. 5). Die PFAS-Analysen in
Bezug auf die Trockensubstanz zeigen hingegen, dass erhöhte Konzentrationen unterhalb der Kläranlage (Felberbach) auftreten (PFOS: $0,01 \mathrm{mg} / \mathrm{kg}$ TS; PFOA: $0,038 \mathrm{mg} / \mathrm{kg}$ TS), mit einem Ausreißer (Probe 1), an dem eine PFOAKonzentration von $0,18 \mathrm{mg} / \mathrm{kg}$ TS festgestellt werden konnte. Anzumerken sind weiters die erhöhten CadmiumWerte $(1,2-2,2 \mathrm{mg} / \mathrm{kg}$ TS) und Bleikonzentrationen $(16,0-29,0 \mathrm{mg} / \mathrm{kg} \mathrm{TS})$, die an allen Probenahmestellen ersichtlich sind.

Die Schwermetallkonzentrationen in Bezug zur organischen Trockensubstanz zeigen ein indifferentes Bild. Die höchsten Belastungen treten an der Probestelle 5 auf, an welcher die Konzentrationen von $\mathrm{Cd}, \mathrm{Hg}$ und $\mathrm{Pb}$ zwischen 63 und $89 \%$ höher liegen (in Relation $\mathrm{zu}$ den übrigen Proben). Im Vergleich zwischen Maltsch und Felberbach können weiters etwas höhere Cadmiumkonzentrationen im Felberbach festgestellt werden (Abb. 3). Die PFAS-Ergebnisse weisen die höchsten gemessenen Konzentrationen unterhalb der Kläranlage im Felberbach auf (Probe 4 und Probe 5). Ein Maximalwert von PFOA $(0,27 \mathrm{mg} / \mathrm{kg}$ oTS $) \mathrm{konn}$ te auch hier in der Probe 1 festgestellt werden.

\section{Diskussion}

Schadstoffe in der aquatischen Umwelt können einen (natürlich) geogen bedingten Hintergrund haben, werden aber durch die anthropogene Nutzung verschiedener Schwermetalle und anorganische sowie organische chemische Verbindungen überlagert (ÖWAV 2013). Die auftretenden Konzentrationen in Fließgewässern werden hierbei durch punktuelle Einleitungen (Kläranlagen, Industrieeinleiter) und diffusen Eintrag (atmosphärische Deposition) beeinflusst (Clara et al. 2014; Schwarzenbach et al. 2010). Für die Bestimmung von Schadstoffen wurden deshalb unterschiedliche Beprobungsmethoden entwickelt, die sich im Wesentlichen durch die Art der (Schadstoff-)Erfassung unterscheiden. Die Entnahme von Wasserproben ist eine einfache Möglichkeit für die Beprobung der Wasserqualität, stellt jedoch immer nur eine Punktmessung dar, wodurch der Entnahmezeitpunkt (jahreszeitliche Schwankungen) einen großen Einfluss auf das (Gesamt-)Ergebnis hat (Clara et al. 2014). Eine automatische Entnahme mittels Pumpen sollte daher ereignisgesteuert erfolgen, 


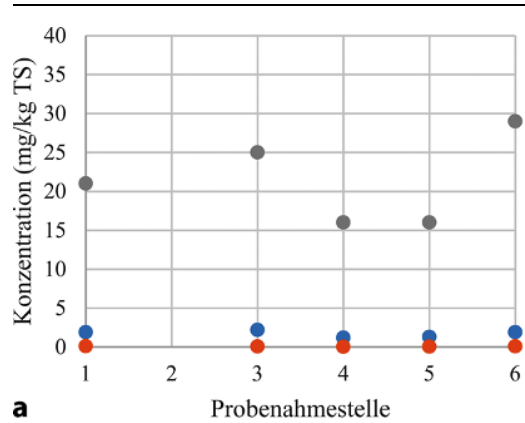

-Cadmium @Quecksilber •Blei

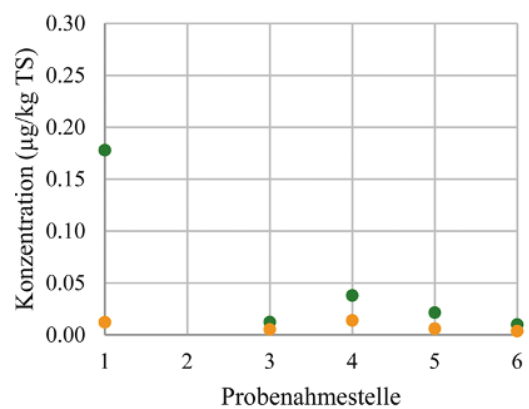

C

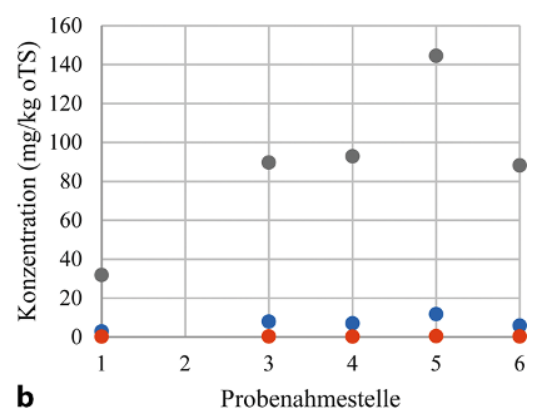

-Cadmium @Quecksilber •Blei

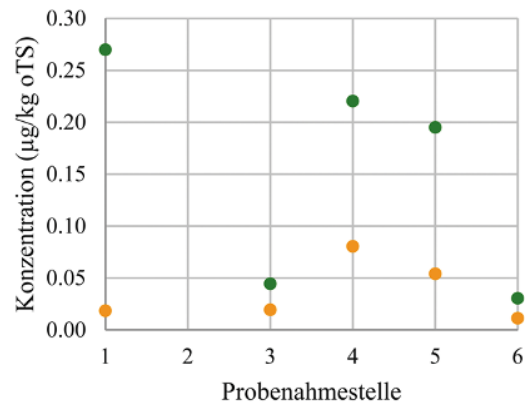

d

-PFOA • PFOS

Abb. 4 EinzugsgebietsbezogeneErgebnissederSpurenstoffe und Schwermetallkonzentrationen bezogen auf die Trockensubstanz $(\mathbf{a}, \mathbf{c})$ und die organische Trockensub$\operatorname{stanz}(\mathbf{b}, \mathbf{d})$
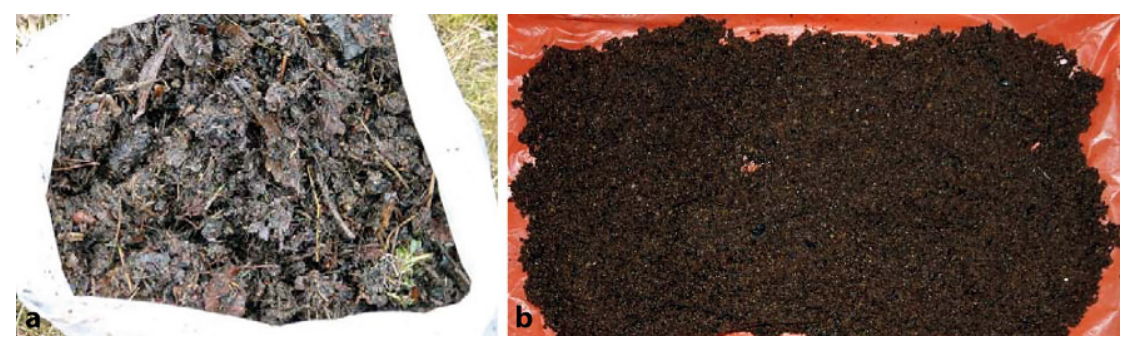

Abb. 5 Beispiele von gesammeltem organischen und anorganischen Material aus dem SOA-Sammler: a überwiegend Blätter und Äste, die in Form von Schwimmstoffen aus der fließenden Welle gesammelt werden (Zubringer durch Forstwirtschaft geprägt); b überwiegend Feinmaterial (Korngrößen $<2 \mathrm{~mm}$ )

erfordert jedoch die nötige Infrastruktur (Stromanschluss, Schutz vor Vandalismus, Wartung etc.). Passive Sammler basierend auf Adsorption und Absorption können hingegen polare und nichtpolare organische Schadstoffe sowie (Schwer-)Metalle in Fließgewässern auch über einen längeren Zeitraum erfassen und finden dadurch breite Anwendung (Booij et al. 2016). Die (oft) schwierige und aufwendige Kalibrierung der Messgeräte ist jedoch wesentlich, um verlässliche Aussagen über die Schadstoffbelastung zu treffen. Weiters wird der Einfluss von Biofouling auf das Messergebnis diskutiert, wodurch eine
Verfälschung nicht ausgeschlossen werden kann (Booij et al. 2006; Guibal et al. 2019). Die Erfassung von Schadstoffkonzentrationen in Schwebstoffen und Kolloiden ist eine weitere Möglichkeit, um auf die Schadstoffbelastung eines Fließgewässers rückzuschließen. Die hierfür entwickelten Messgeräte eignen sich sehr gut für mittlere bis größere Fließgewässer mit ausreichender Strömungsgeschwindigkeit (u.a. Draxler et al. 2012; Phillips et al. 2000). Bei zu geringen Fließgeschwindigkeiten können auch mittels zugeschalteter Pumpen gute Proben entnommen werden, benötigen aber auch hier die zusätzli- che Infrastruktur (geeigneter Standort, Strom etc.). Der in diesem Beitrag vorgestellte stationäre Organik- und Anorganik-Sammler (SOAS) basiert auf dem Prinzip von Bunte et al. (2007), bei dem Geschiebe, Schweb-, und Schwimmstoffe in einem Netz aufgefangen werden. Durch die deutliche Reduktion der Netzgröße auf 0,5 $\mathrm{mm}$ wird der von Bunte et al. (2007) beschriebene Effekt der Netzverlegung (mechanisch, biologisch) genutzt, um Schweb-, und Schwimmstoffe aus der fließenden Welle zu erfassen. Dies hat den Vorteil, dass auch bei geringen Fließgeschwindigkeiten und (erhöhter) Nährstoffbelastung ausreichend Material gesammelt werden kann. Die Ergebnisse dieser ersten Untersuchung im Maltsch-Einzugsgebiet (Oberösterreich) zeigen hierbei das Potenzial der Beprobung auf Spurenstoffe und Schwermetalle in watbaren Fließgewässern mit kleinen Fließgeschwindigkeiten, da die (Bio-)Kolmatierung der Netze die Sammelleistung nicht beeinträchtigt haben.

Gezeigt werden konnten bei dieser Untersuchung auch Unterschiede zwischen Sedimentablagerungen (unbekannte Expositionszeit) und den Proben aus dem SOA-Sammler (Material über 2 Wochen gesammelt). Die einzugsgebietsbezogenen Ergebnisse zeigen hierbei nicht nur Unterschiede in den Schadstoffkonzentrationen in Bezug auf die organische und anorganische Trockensubstanz, sondern auch eine einzugsgebietsbezogene Variabilität. Aufgrund der geringen Anzahl an Proben kann zwar kein (genereller) Trend beschrieben werden, es zeigen sich jedoch einige interessante stoffspezifische Unterschiede. Die Quecksilberund Bleikonzentration der Trockensubstanz weisen ähnliche Werte wie in bereits veröffentlichten Studien aus Österreich auf (Amann et al. 2019). Hingegen sind die Cadmiumkonzentrationen im Maltsch-Einzugsgebiet auffallend. Im Geochemischen Atlas Österreichs (Pirkl et al. 2015) werden zwar keine flächigen Hintergrundinformationen zur Cadmiumbelastung in Oberösterreich ausgewiesen, jedoch wird darauf hingewiesen, dass granitisches Gestein eine erhöhte Cadmium-Häufigkeit $\left(0,2^{2}\right.$ ppm) aufweist. Auch für Blei wird hier eine deutlich höhere Häufigkeit in granitischem Gestein (20 20 pm) ausgewiesen. Eine natürliche, geogene Belastung wird daher als wahrscheinlich angenommen. Die einzugsgebietsbezogenen Analysen der PFAS-Gruppe 
weisen deutlich geringere PFOS-Konzentrationen (bezogen auf die Trockensubstanz) wie in bereits publizierten Studien auf (Amann et al. 2019). Im Maltsch-Oberlauf (Probe 1) konnte hingegen die höchste PFOA-Konzentration festgestellt werden $(0,18 \mathrm{mg} / \mathrm{kg} \mathrm{TS})$, während die übrigen Probenkonzentrationen unter $0,04 \mathrm{mg} / \mathrm{kg}$ TS aufwiesen. Der diffuse Eintrag über atmosphärische Deposition könnte daher den maßgebenden Eintragspfad darstellen, da in diesem Abschnitt der Maltsch keine Punktquelle bekannt ist.

Die ersten Analysen im Einzugsgebiet der Maltsch zeigen die gute Anwendbarkeit dieses Sammlers für kleine Fließgewässer. Insbesondere durch die einfache Handhabung und Erfassung aller transportierten Stoffe (Blätter, Äste, Schwebstoffe etc.) können Schadstoffkonzentrationen in Schwebstoffen von Gewässern mit kleinen Einzugsgebieten erfasst werden (Abb. 5). Das vorgestellte Konzept stellt dadurch eine gute Ergänzung zu den bekannten Probenahmemethoden dar.

\section{Schlussfolgerungen und Anwendungsgebiete}

Im vorliegenden Beitrag wird ein Konzept zur Sammlung von organischem und anorganischem Material in watbaren Fließgewässern vorgestellt. Durch die geringe Maschenweite $(0,5 \mathrm{~mm})$ des adaptierten Geschiebefängers von Bunte et al. (2007) kann innerhalb eines Tages die gewünschte (Bio-)Kolmation festgestellt werden, die eine Sammlung von Schweb- und Schwimmstoffen begünstigt. Dies ist insofern von Bedeutung, als dass bei bereits bekannten passiven Wasser- und Sedimentqualitäts-Beprobungsmethoden die (Bio-)Kolmation oft eine Limitierung darstellt, wenn im jeweiligen Gewässer- abschnitt hohe Nährstoffbelastungen und geringe Fließgeschwindigkeiten auftreten. Weiters hat sich gezeigt, dass durch das Sammelkonzept Schwebund Schwimmstoffe in ausreichend großer Menge gesammelt werden können, um organische und anorganische Schadstoffe nachweisen zu können. Hierbei zeigen sich bereits deutliche Unterschiede in den Schadstoffkonzentrationen kleiner (Teil-)Einzugsgebiete $\left(<100 \mathrm{~km}^{2}\right)$, wenn das Material über einen längeren Zeitraum in strömungsberuhigten Zonen im Gewässer ablagert oder nur über einen begrenzten Zeitraum gesammelt wird.

Der Fokus bisheriger Studien in Österreich lag in erster Linie auf größere Flusseinzugsgebiete (z.B. Donau, Drau, Inn), Fließgewässer mit erhöhtem (diffusen) Nährstoffeintrag oder auf Untersuchungen im Hinblick auf ökologische Folgen durch Punktquellen (Kläranlagen, Industrieeinleiter). Die vorgestellten Ergebnisse zeigen jedoch das Forschungspotenzial im Bereich des Schadstoffeintrags und -transports sowie deren Ab- und Umbau in der aquatischen Umwelt in kleinen Einzugsgebieten $\left(<100 \mathrm{~km}^{2}\right)$. Im Zuge des Christian-Doppler-Labors für Sedimentforschung und -management sollen diese Aspekte daher weiter erforscht und aufgezeigt werden.

Danksagung Wir bedanken uns für die finanzielle Unterstützung durch die Christian-Doppler-Forschungsgesellschaft, das Bundesministerium für Digitalisierung und Wirtschaftsstandort und die Nationalstiftung für Forschung, Technologie und Entwicklung. Darüber hinaus möchten wir unseren Unternehmenspartnern Verein für Ökologie und Umweltforschung (VÖU), viadonau, Andritz AG und Voith $\mathrm{GmbH}$ für die Förderung unserer Forschungsarbeiten im Rahmen des CD-Labors danken. Die Autoren möchten sich ebenfalls beim Auftraggeber (Amt der OÖ. Landesregierung) für die Initiierung und Finanzierung der Studie „Förderung der natürlichen Umwelt und des Vorkommens der Flussperlmuschel im Maltsch-Einzugsgebiet" bedanken, ebenso wie für die finanzielle Unterstützung durch den Europäischen Fonds für regionale Entwicklung (Interreg-Förderprogramm).

Funding Open access funding provided by University of Natural Resources and Life Sciences Vienna (BOKU).

Open Access Dieser Artikel wird unter der Creative Commons Namensnennung 4.0 International Lizenz veröffentlicht, welche die Nutzung, Vervielfältigung, Bearbeitung, Verbreitung und Wiedergabe in jeglichem Medium und Format erlaubt, sofern Sie den/die ursprünglichen Autor(en) und die Quelle ordnungsgemäß nennen, einen Link zur Creative Commons Lizenz beifügen und angeben, ob Änderungen vorgenommen wurden.

Die in diesem Artikel enthaltenen Bilder und sonstiges Drittmaterial unterliegen ebenfalls der genannten Creative Commons Lizenz, sofern sich aus der Abbildungslegende nichts anderes ergibt. Sofern das betreffende Material nicht unter der genannten Creative Commons Lizenz steht und die betreffende Handlung nicht nach gesetzlichen Vorschriften erlaubt ist, ist für die oben aufgeführten Weiterverwendungen des Materials die Einwilligung des jeweiligen Rechteinhabers einzuholen.

Weitere Details zur Lizenz entnehmen Sie bitte der Lizenzinformation auf http://creativecommons.org/licenses/ by/4.0/deed.de.

\section{Literatur}

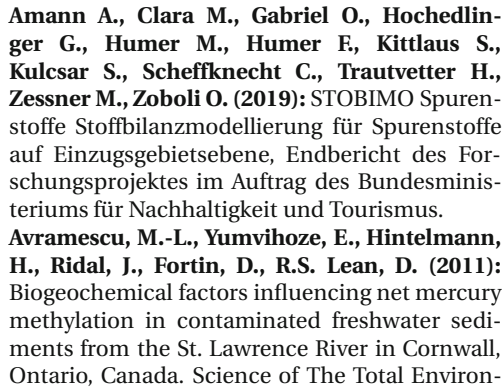

Amann A, Clara M. Gabriel O, Hochedlinger G., Humer M., Humer F., Kittlaus S. Kulcsar S., Scheffknecht C., Trautvetter H. Zessner M., Zoboli O. (2019): STOBIMO Spurenstoffe Stoffbilanzmodellierung für Spurenstoffe auf Einzugsgebietsebene, Endbericht des Forschungsprojektes im Auftrag des Bundesministeriums für Nachhaltigkeit und Tourismus. Avramescu, M.-L., Yumvihoze, E., Hintelmann, H., Ridal, J., Fortin, D., R.S. Lean, D. (2011) Biogeochemical factors influencing net mercury methylation in contaminated freshwater sediments from the St. Lawrence River in Cornwall, Ontario, Canada. Science of The Total Environ-

ment 409, 968-978. https://doi.org/10.1016/j. scitotenv.2010.11.016

Booij, K., van Bommel, R., Mets, A., Dekker, R. (2006): Little effect of excessive biofouling on the uptake of organic contaminants by semipermeable membrane devices. Chemosphere 65, 2485-2492. https://doi.org/10.1016/j. chemosphere.2006.04.033

Booij, K., Robinson, C.D., Burgess, R.M., Mayer,

P., Roberts, C.A., Ahrens, L., Allan, I.J., Brant,

J., Jones, L., Kraus, U.R., Larsen, M.M., Lepom,

P., Petersen, J., Pröfrock, D., Roose, P., Schäfer

S., Smedes, F., Tixier, C., Vorkamp, K., White-

house, P. (2016): Passive Sampling in Regulatory

Chemical Monitoring of Nonpolar Organic Com- pounds in the Aquatic Environment. Environ Sci. Technol. 50, 3-17. https://doi.org/10.1021/ acs.est.5b04050

Bunte, K., Swingle, K.W., Abt, S.R. (2007): Guidelines for using bedload traps in coarsebedded mountain streams: Construction, installation, operation, and sample processing, Gen. Tech. Rep. RMRS-GTR-191. U.S. Department of Agriculture, Forest Service, Rocky Mountain Research Station, Fort Collins, CO.

Clara, M., Ertl, T., Giselbrecht, G., Gruber, G. Hofer, T., Humer, F., Kretschmer, F., Kolla, L., Scheffknecht, C., Weiß, S., Windhofer, G. (2014): Spurenstoffemissionen aus Siedlungsgebieten und von Verkehrsflächen. Studie im 
Auftrag des Bundesministeriums für Land- und Forstwirtschaft, Umwelt und Wasserwirtschaft, Wien, Österreich.

Draxler, A., Gans, O., Humer, F., Vrana, B. (2012): HESTIA - Harmonisierung und Evaluierung von Probenahmetechniken in der aquatischen Umwelt. Federal Ministry of Agriculture, Forestry, Environment and Water Management, Vienna, Austria.

Förstner, U. (2004): Sediment dynamics and pollutant mobility in rivers: An interdisciplinary approach. Lakes \& Reservoirs: Science, Policy and Management for Sustainable Use 9, 25-40. https://doi.org/10.1111/j.1440-1770 2004.00231.x

Garcia-Ordiales, E., Loredo, J., Covelli, S., Esbri, J.M., Millan, R., Higueras, P. (2017): Trace metal pollution in freshwater sediments of the world's largest mercury mining district: sources, spatia distribution, and environmental implications. J. Soils Sediments 17, 1893-1904. https://doi.org/ 10.1007/s11368-016-1503-5

Guibal, R., Buzier, R., Lissalde, S., Guibaud, G (2019): Adaptation of diffusive gradients in thin films technique to sample organic pollutants in the environment: An overview of o-DGT passive samplers. Science of The Total Environmen 693, 133537. https://doi.org/10.1016/j.scitotenv. 2019.07.343

Haitzer, M., Höss, S., Traunspurger, W., Steinberg, C. (1998): Effects of dissolved organic matter (DOM) on the bioconcentration of organic chemicals in aquatic organisms - a reviewChemosphere 37, 1335-1362. https://doi.org/10 1016/S0045-6535(98)00117-9

Hjulström, F. (1935): Studies of the morphological activity of rivers as illustrated by the River Fyris: Inaugural Dissertation. Almqvist \& Wiksells, Uppsala.
Inostroza, P.A., Massei, R., Wild, R., Krauss, M. Brack, W. (2017): Chemical activity and distribution of emerging pollutants: Insights from a multi-compartment analysis of a freshwater system. Environ. Pollut. 231, 339-347.https://doi.org/10. 1016/j.envpol.2017.08.015

Järup, L. (2003): Hazards of heavy metal contamination. Br Med Bull 68, 167-182. https://doi. org/10.1093/bmb/ldg032

Malaj, E., Ohe, P.C. von der, Grote, M., Kühne, R., Mondy, C.P., Usseglio-Polatera, P., Brack, W. Schäfer, R.B. (2014): Organic chemicals jeopardize the health of freshwater ecosystems on the continental scale. PNAS 111, 9549-9554. https:// doi.org/10.1073/pnas.1321082111

Ouddane, B., Monperrus, M., Kadlecova, M. Daye, M., Amouroux, D. (2014): Mercury methylation and demethylation in highly contaminated sediments from the Deûle River in Northern France using species-specific enriched stable isotopes. Environ. Sci.: Processes Impacts 17, 145-155. https://doi.org/10.1039/ C4EM00398E

ÖWAV (2013): Anthropogene Spurenstoffe in der aquatischen Umwelt, Positionspapiere des Österreichischen Wasser- und Abfallwirtschaftsverbandes (ÖWAV). Österreichischer Wasserund Abfallwirtschaftsverband, Wien.

Phillips, J.M., Russell, M.A., Walling, D.E. (2000): Time-integrated sampling of fluvial suspended sediment: a simple methodology for small catchments. Hydrological Processes 14, 2589-2602. https://doi.org/10.1002/10991085(20001015) 14:14<2589::AID-HYP94>3.0. CO;2-D

Pirkl, H., Schedl, A., Pfleiderer, S. (Hrsg.) (2015): Geochemischer Atlas von Österreich - Bundesweite Bach- und Flusssedimentgeoche- mie (1978-2010). - Archiv für Lagerstättenforschung, 28, 288 S., Wien.

Schwarzenbach, R.P., Egli, T., Hofstetter, T.B. von Gunten, U., Wehrli, B. (2010): Global Water Pollution and Human Health. Annual Review of Environment and Resources 35, 109-136. https://doi.org/10.1146/annurevenviron-100809- 125342

Shinn, C., Dauba, F., Grenouillet, G., Guenard, G., Lek, S. (2009): Temporal variation of heavy metal contamination in fish of the river lot in southern France. Ecotoxicol. Environ. Saf. 72, 1957-1965. https://doi.org/10.1016/j.ecoenv. 2009.06.007

Zessner, M., Zoboli, O., Reif, D., Amann, A. Sigmund, E., Kum, G., Saracevic, Z., Saracevic, E., Kittlaus, S., Krampe, J. (2019): Belastung des Neusiedler Sees mit anthropogenen Spurenstoffen: Überlegungen zu Herkunft und Verhalten. Österreichische Wasser-und Abfallwirtschaft 1-15. https://doi.org/10.1007/s00506019-00623-1

Zoboli, O., Clara, M., Gabriel, O., Scheffknecht,

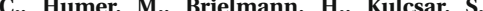
Trautvetter, H., Kittlaus, S., Amann, A., Saracevic, E., Krampe, J., Zessner, M. (2019): Occurrence and levels of micropollutants across environmental and engineered compartments in Austria. Journal of Environmental Management 232, 636-653. https://doi.org/10.1016/j. jenvman.2018.10.074

Hinweis des Verlags Der Verlag bleibt in Hinblick auf geografische Zuordnungen und Gebietsbezeichnungen in veröffentlichten Karten und Institutsadressen neutral. 